\title{
Lack of association in acne and salivary testosterone
}

\author{
David Fresnais ${ }^{1}$, Edvin Ingberg², Elvar Theodorsson ${ }^{3}$, Jakob O. Ström ${ }^{1,3}$
}

${ }^{1}$ Department of Neurology, Faculty of Medicine and Health, Örebro University, Örebro, Sweden; ${ }^{2}$ Department of Infection, Faculty of Medicine and Health, Örebro University, Örebro, Sweden; ${ }^{3}$ Department of Clinical Chemistry and Department of Clinical and Experimental Medicine, Linköping University, Linköping, Sweden

\section{Abstract}

The pathogenesis of acne vulgaris has only been partially elucidated. Various hormones, especially androgens, are likely to play a role, but results of studies are still inconclusive. The objective of the current study was to investigate whether day to day variation in salivary testosterone correlates with acne in males. Saliva samples were collected for 120 consecutive days from each of the 40 males. Salivary testosterone concentrations were measured by enzyme-linked immunosorbent assay (ELISA). Facial acne lesions were assessed on a daily basis by photography by the participating males. Potential confounders' (sexual intercourse, masturbation, physical exercise and disease) were also registered every day by the participants. A significant but weak association between salivary testosterone and acne was found $(n=4602, r=0.031, P=0.034)$. Elevated testosterone concentrations were associated with an increase in acne, but when testosterone concentrations were above twice the individual average, acne lesions paradoxically decreased. The current results indicate that daily fluctuations in salivary testosterone levels in males are associated with acne patterns, but the weak correlation suggests that the effect is too small to be of clinical significance. The analysis in the current study was complicated by a large number of days on which the participants had no acne, as well as the seemingly non-monotonic relation between testosterone and acne. This may indicate that the actual relation is stronger than concluded here.

Keywords: acne; androgens; male; saliva; testosterone

Received: 17 April 2018; Accepted after revision: 31 May 2018; Published: 09 July 2018

Author for Correspondence: Jakob O Ström, Department of Neurology, Örebro University Hospital, 70185 Örebro, Sweden. Email: jakob.strom@ oru.com

How to cite: Fresnais D et al. Lack of association between fluctuations in acne and salivary testosterone. J Controversies Biomed Res. 2018;4(1):1-5

Doi: http://dx.doi.org/10.15586/jcbmr.2018.25

Copyright: Fresnais D et al.

License: This open access article is licensed under Creative Commons Attribution 4.0 International (CC BY 4.0). http://creativecommons.org/ licenses/by/4.0

\section{Introduction}

Acne is a non-infectious, chronic inflammation of the pilosebaceous unit (1). It is the most common skin disease affecting at least $85 \%$ of adolescents (2). The pathogenesis of acne vulgaris is incompletely understood and is currently attributed to multiple factors. At least four factors have been included to have a role in its development: hyperkeratinization of the sebaceous follicles and development of a keratin plug that may obstruct the outflow of the sebum to the skin surface, increased sebum production, microbial hypercolonization of the pilosebaceous units by Propionibacterium acnes, and secondary follicular inflammation (1).
Both clinical and experimental evidence support the role of androgens in stimulating sebum production: (i) the presence of acne in the prepubertal period correlates with serum levels of dehydroepiandrosterone sulfate (DHEAS), an adrenal precursor for synthesis of testosterone $(3,4)$, (ii) subjects who lack functional androgen receptors do not produce sebum, and consequently do not develop acne (3), (iii) tumors of the ovary or the adrenal gland that are androgen producing are often associated with the development of acne (3), (iv) systemic administration of testosterone and DHEAS increases the size of the sebaceous glands and stimulates sebum production $(3,5),(v)$ severe acne is 
often associated with conditions of hyperandrogenism and increased sebum production $(2,3,5)$. It has also been suggested that hormones affect hyperkeratinization of the sebaceous follicles (5).

However, the results regarding the role of testosterone in the pathogenesis of acne vulgaris are still inconclusive. Ramsay et al. found that free testosterone levels were elevated in men with acne vulgaris compared to an age-matched control group (6). Yang et al. reported an increase in testosterone levels in women with acne but not in males (7). In contrast, Schmidt et al. showed that men with acne had increased cortisol levels but normal testosterone levels (8) and Placzek et al. compared the levels of testosterone, luteinizing hormone, follicle-stimulating hormone, DHEAS, androstenedione, and 17-hydroxyprogesterone between men with and without acne, finding that only 17-hydroxyprogesterone was significantly elevated in men with acne (9).

We hypothesized that if testosterone was important in acne pathophysiology, daily fluctuations in testosterone would be reflected in acne variations. The current study was therefore initiated to test this hypothesis by comparing daily male salivary testosterone concentrations with the corresponding daily acne numbers.

\section{Materials and Methods}

Before participant recruitment, the study protocol was approved by the Regional Ethical Review Board of Uppsala (2014/360). The study was performed in accordance with relevant guidelines and regulations. Informed consent was obtained from all participants. This study was part of a larger project studying the effect of social conditions on testosterone concentrations, aiming to include 48 heterosexual couples. Participants were recruited by posters and flyers on the university campuses in Linköping (Sweden) and Örebro (Sweden) and by an advertisement in the local newspaper in Örebro. The posters, flyers, and advertisement specified that the participants must be $18-50$ years of age. After the participants contacted the research team, they were provided more detailed information about the study and asked to answer an inclusion/exclusion questionnaire. The inclusion was: at least 18 years old. Exclusion criteria were: (a) previous gonadectomy, (b) disease that could affect the sex hormone system, (c) consumption of prescribed drugs that could alter the sex hormone system and (d) consumption of anabolic steroids.

Before commencing the study, at least one person from each included couple attended a start-up meeting with the research team for detailed practical instructions. Fifty couples were finally included and 48 completed the study. Forty of the males reported at least one acne lesion and were included in the current study. For participating in the study, each male participant received the SEK equivalent of $€ 300$.

\section{Saliva samples}

The study proceeded for 120 days. Saliva samples were taken at home the same time every morning by the participating males (Salivette test tubes with synthetic oral swabs, product no. 51.1534.500, Sarstedt AG \& Co, Nümbrecht, Germany). Any temporal deviations were registered in a protocol, but to avoid such errors all male participants were offered to receive a text message reminder on their cellular phones the same time every morning. Participants were told not to eat or brush their teeth prior to sampling. The saliva samples were immediately put in a plastic bag and stored in the home freezer until the end of the sampling period. After the sampling period, all samples were collected by the research team and stored in $-80^{\circ} \mathrm{C}$ until analysis. The other outcome variable, acne, was registered daily by each male participant. To enable inter-individual comparison, only acne lesions in the face, from forehead to the lower edge of the mandible, and in front of the ears, were counted.

In earlier studies, intense physical exercise (10), sexual activity $(11,12)$, and illness $(13)$ have been reported to acutely influence testosterone levels. To control for these factors, all males received protocols in which they were instructed to record time of masturbation, intercourse (defined as sexual activity leading to male orgasm, with involvement of the partner), training (defined as physical activity with the intent of training), illness (defined as infection rendering fever or other illness preventing the participant to go to work, had it been a working-day), absence from partner overnight and sampling miss-happenings (such as forgetting to put the sample in the freezer or eating prior to sampling).

\section{Testosterone analysis}

Saliva samples were centrifuged for $15 \mathrm{~min}$ at $1500 \mathrm{~g}$ and analyzed by testosterone enzyme immunoassay (DES6622, Demeditec Diagnostics GmbH, Kiel, Germany) according to the manufacturer's protocol.

\section{Statistical analyses}

The outcomes of salivary testosterone and acne were assessed in parallel as follows:

Mean values for the entire study's salivary testosterone and acne numbers were calculated for each male participant. Then each daily salivary testosterone concentration and acne number was divided by the individual's mean value, for inter-individual standardization. Hence, if a specific day had a standardized salivary testosterone concentration of, for example, 2, it meant that the individual on that day had twice as high testosterone concentration as usual.

IBM SPSS statistics 20 was used for all analyses. First, a multiple regression analysis was performed to assess whether the potential confounders-sexual intercourse the day before, masturbation the day before, training the day before, 
and disease the same day - affected standardized acne numbers. Since no significant effect of the potential confounders was found, the main analysis was limited to a Pearson correlation analysis between standardized salivary testosterone and standardized acne. Further, the correlation between acne and testosterone levels the day before, 2 days before, 3 days before, 4 days before, and 1 day after was analyzed.

As a further ad hoc analysis to investigate if the change of acne from one day to another was more closely associated to testosterone, another correlation analysis was performed. In this analysis, an acne change value was tested for correlation to standardized testosterone the same day. The acne change value was calculated by subtracting the number of acne lesions of the preceding day from the current day's value. Hence, if a specific day had an acne number of 2 and the number was 3 the day before, the acne change value would be $-1(2,3)$.

\section{Results}

Of the 50 males who initially signed up for the study, 40 had at least one acne lesion during the 120-day period, 8 were excluded from the analyses because they did not report any acne lesions, and 2 did not complete the study. The mean age of the 40 participating males was 31.46 with a standard deviation of 6.983 years. None of the potential confounders significantly affected standardized acne $(n=4560$; sexual intercourse, $P=0.454$; masturbation, $\mathrm{P}=0.783$; training, $\mathrm{P}=0.622$; disease, $\mathrm{P}=0.072$ ).

In the main analysis - without confounder control - there was a statistically significant correlation between the standardized number of acne lesions and salivary testosterone levels $(\mathrm{n}=4602, \mathrm{r}=0.031, \mathrm{P}=0.034)$. As seen in Table 1, standardized acne increased with standardized testosterone to a value of 2 on the latter, to subsequently decrease, indicating a non-monotonic relation.

There was no significant correlation between the number of acne lesions and the salivary testosterone levels recorded the day before $(\mathrm{n}=4566, \mathrm{r}=0.026, \mathrm{P}=0.082), 2$ days before $(\mathrm{n}=4532, \mathrm{r}=0.003, \mathrm{P}=0.865), 3$ days before $(\mathrm{n}=4497$, $r=-0.006, P=0.673), 4$ days before $(n=4460, r=0.022$, $\mathrm{P}=0.143)$, or 1 day after $(\mathrm{n}=4563, \mathrm{r}=0.022, \mathrm{P}=0.129)$.

No significant correlations were found between acne change (difference between the acne number a specific day and the day before) and standardized testosterone ( $\mathrm{n}=3909$, $r=-0.004, P=0.794)$.

\section{Discussion}

By analyzing the salivary testosterone concentrations in male subjects and comparing them to self-reported acne lesions, we found that variations in day to day testosterone levels were associated with acne variations, but the correlation was very low $(r=0.031)$. However, this low $r$-value means that salivary testosterone explained merely $0.1 \%$ of the variation in acne numbers, making the association clinically insignificant.
Table 1. Intervals of standardized testosterone and the corresponding average standardized acne values.

\begin{tabular}{|l|c|c|}
\hline $\begin{array}{l}\text { Standardized } \\
\text { testosterone interval }\end{array}$ & $\begin{array}{c}\text { Average } \\
\text { standardized acne }\end{array}$ & $\begin{array}{c}\text { Number of } \\
\text { observations }\end{array}$ \\
\hline $0-0.24$ & 0.69 & 6 \\
\hline $0.25-0.49$ & 0.45 & 181 \\
\hline $0.5-0.74$ & 0.89 & 903 \\
\hline $0.75-0.99$ & 0.95 & 1545 \\
\hline $1-1.24$ & 1.18 & 1112 \\
\hline $1.25-1.49$ & 1.19 & 485 \\
\hline $1.5-1.74$ & 1.42 & 202 \\
\hline $1.75-1.99$ & 1.88 & 83 \\
\hline $2-2.24$ & 0.63 & 36 \\
\hline $2.25-2.49$ & 1.00 & 18 \\
\hline $2.5-2.74$ & 0.36 & 10 \\
\hline $2.75-2.99$ & 0.25 & 7 \\
\hline $3+$ & 0.56 & 15 \\
\hline
\end{tabular}

Because acne vulgaris is a multifactorial disease, there are many potential reasons as to why testosterone levels had a slight effect on acne variations. Androgens have been shown to stimulate DNA-synthesis in the epidermis by impairing the barrier function of the skin, which results in epidermal hyperplasia and follicular hyperkeratosis (14). This as well as other factors covered in the introduction section are likely to contribute.

One possible explanation to the weak association is that fluctuations in circulating testosterone may not significantly affect the local hormone concentration in the skin. Studies have shown that the skin, as well as the sebaceous glands, has the ability to produce and metabolize androgens, which could abate the association between salivary testosterone and acne. Dehydroepiandrosterone (DHEA) sulfate is the major adrenal precursor for more potent androgens, including testosterone and dihydrotestosterone. All enzymes necessary for the conversion of cholesterols to steroids, DHEA sulfate, and DHEA are locally present in the skin, and the enzymes required for converting DHEA to androstenedione and testosterone have been localized to the sebaceous gland (15). Although androgens seem to have a role in acne pathogenesis, it is uncertain whether the cause is circulating androgens or androgens produced locally in the skin, or a combination of both (3).

Another explanation to the relatively weak correlation coefficient could be that males respond differently to androgen fluctuations, with responders and non-responders. In that 
case, a potential association could be lost, especially if nonresponders were more common than responders. It is worth considering that many men do not have acne, despite having relatively high levels of androgens compared with women. This could also suggest a gender difference in the hormonal aspects of acne pathogenesis. In addition, many individuals with high sebum production do not develop acne (4).

A further possible explanation for the weak association can be found in Table 1, in which it is illustrated that the correlation between acne and testosterone does not seem to be linear, but rather shaped like an inverted ' $U$ '. Initially rising testosterone levels were associated with an increased number of acne lesions, but as standardized testosterone levels rose to a value of 2 (indicating twice the daily average for that individual) or greater, acne lesions decreased. We interpret this surprising finding as an indication that the dose-response relation between testosterone may be non-monotonic. Hence, the method used for analyzing the material-linear regression - is sub-optimal because it focuses on linear correlations. Consequently, it is likely that the correlation between acne and testosterone is stronger than our results suggest. Existing literature cannot provide a satisfying explanation to this non-monotonic dose-response phenomenon, because it has not been observed before. We can only speculate that when circulating testosterone levels increase to a certain extent, it affects the sebaceous glands differently or interferes with the production or binding of other androgens to key receptors in the skin.

Before the study was conducted, we suspected that there may be a delay between the circulating testosterone concentrations and its effect on acne, so that the number of acne lesions would be dependent on the testosterone concentrations from previous days. However, the relation between testosterone and acne was not improved by time-wise skewing of the analysis up to 4 days. This suggests that the daily fluctuations in free testosterone levels affected, even if only slightly, acne patterns observed during the same day.

In earlier studies, intense physical exercise, sexual activity, and illness have been reported to influence testosterone levels, which is why they were considered potential confounders in the current study. The multiple regression analysis did not show any significant correlations between any of these parameters. It appeared to be a weak correlation between acne and illness but not strong enough to reach statistical significance $(\mathrm{P}=0.072)$. Therefore, the variables were not included as confounder controls in the main analysis.

To our best knowledge, there are no similar studies in the literature, but several case-control studies with conflicting results have been published. In three studies, males with acne had higher levels of 17-hydroxyprogesterone (9), cortisol (8), and estradiol (7), respectively, but not testosterone, which is in line with the current study. On the contrary, Ramsay et al. found that male acne patients had higher testosterone levels than controls, indicating a possible contribution of testosterone in acne pathophysiology. In addition, testosterone levels were high in females with acne in two studies $(7,8)$. All of the abovementioned studies were relatively small in terms of the number of analyzed samples and therefore potentially underpowered. This could explain the discrepant results, even more if the effect of testosterone on acne is very small, as suggested by the current study.

\section{Limitations}

Excess sebum causes comedones which are papules with white or black heads. In this study, for convenience, only inflammatory acne lesions were counted, which may be seen as a weakness. Subjects who did not report any acne lesions during the study were excluded from the statistical analyses. Still there were many subjects who reported only one or two acne lesions during the entire duration of the study. Linear regression analysis is not optimal on datasets with a high proportion of zero values, which may have resulted in an underestimation of the testosterone-acne association. Further, when standardizing the acne results to each individual's mean value, individuals with very low average acne numbers got a few very high standardized values. These high values might have affected the results by having a disproportionally high impact in the analysis. To assess the importance of this potential bias, a linear regression analysis was re-performed with exclusion of standardized acne values exceeding 20, and the previously found correlation was lost $(\mathrm{P}=0.460, \mathrm{r}=0.059, \mathrm{n}=4560)$. This calls for even more careful interpretation of the results. A further limitation of the current study was that several thousands of observations from only 40 patients were analyzed as independent observations in the linear regression analyses, which obviously is not strictly correct. This was the reason for making the individual standardization prior to the acne and testosterone values prior to the regression analyses.

\section{Conclusion}

The current results indicate that circulating testosterone may have a slight influence on acne variations in males, and appears to affect acne patterns the same day. A rise in testosterone is associated with an increase in acne when standardized testosterone levels are below 2 (twice the daily average for that individual), whereas at greater levels acne paradoxically appear to decrease. However, these findings have no clinical significance since circulating testosterone levels explained approximately $0.1 \%$ of the observed acne variations.

\section{Acknowledgements}

The study was funded by Linköping University, Örebro University, Region Östergötland and Region Örebro Län (Forskningskommittén). 


\section{Conflict of interest}

J.O. Strom received consultant fee from Bayer AB in 2016 for participation in an advisory board regarding anticoagulants and atrial fibrillation. Other authors declare no conflict of interest with respect to research, authorship, and/or publication of this article.

\section{References}

1. Thiboutot D, Gollnick H, Bettoli V, Dréno B, Kang S, Leyden $\mathrm{JJ}$, et al. New insights into the management of acne: An update from the Global Alliance to Improve Outcomes in Acne group. J Am Acad Dermatol. 2009;60(5 Suppl):S1-50.

2. Arora MK, Yadav A, Saini V. Role of hormones in acne vulgaris. Clin Biochem. 2011;44(13):1035-40. http://dx.doi.org/10.1016/j. clinbiochem.2011.06.984

3. Thiboutot D. Acne: Hormonal concepts and therapy. Clin Dermatol. 2004;22(5):419-28. http://dx.doi.org/10.1016/j.clindermatol.2004.03.010

4. Thiboutot D, Gilliland K, Light J, Lookingbill D. Androgen metabolism in sebaceous glands from subjects with and without acne. Arch Dermatol. 1999;135(9):1041-5. http://dx.doi. org/10.1001/archderm.135.9.1041

5. Bakry OA, El Shazly RMA, El Farargy SM, Kotb D. Role of hormones and blood lipids in the pathogenesis of acne vulgaris in non-obese, non-hirsute females. Indian Dermatol Online J. 2014;5(Suppl 1):S9-16. http://dx.doi.org/10.4103/ 2229-5178.144506

6. Ramsay B, Alaghband-Zadeh J, Carter G, Wheeler MJ, Cream JJ. Raised serum androgens and increased responsiveness to luteinizing hormone in men with acne vulgaris. Acta Derm Venereol. 1995;75(4):293-6.

7. Yang XQ, Shen XL, Wu ER, Xia TA. Testosterone and estradiol serum levels in acne. Chin Med J (Engl). 1989;102(3): 236-8.

8. Schmidt JB, Lindmaier A, Spona J. Endocrine parameters in acne vulgaris. Endocrinol Exp. 1990;24(4):457-64.

9. Placzek M, Arnold B, Schmidt H, Gaube S, Keller E, Plewig $\mathrm{G}$, et al. Elevated 17-hydroxyprogesterone serum values in male patients with acne. J Am Acad Dermatol. 2005;53(6):955-8. http://dx.doi.org/10.1016/j.jaad.2005.07.014

10. Vermeulen A. Testosterone in plasma. A physiopathological study. Verh - K Acad Voor Geneeskd Van Belg. 1973;35(2):95-180.

11. Hirschenhauser K, Frigerio D, Grammer K, Magnusson MS. Monthly patterns of testosterone and behavior in prospective fathers. Horm Behav. 2002;42(2):172-81. http://dx.doi.org/ 10.1006/hbeh.2002.1815

12. Purvis K, Landgren BM, Cekan Z, Diczfalusy E. Endocrine effects of masturbation in men. J Endocrinol. 1976;70(3):439-44. http://dx.doi.org/10.1677/joe.0.0700439

13. Allen JA, Diemer T, Janus P, Hales KH, Hales DB. Bacterial endotoxin lipopolysaccharide and reactive oxygen species inhibit Leydig cell steroidogenesis via perturbation of mitochondria. Endocrine. 2004;25(3):265-75. http://dx.doi.org/10.1385/ ENDO:25:3:265

14. Seirafi H, Farnaghi F, Vasheghani-Farahani A, Alirezaie N-S, Esfahanian F, Firooz A, et al. Assessment of androgens in women with adult-onset acne. Int J Dermatol. 2007;46(11):1188-91. http://dx.doi.org/10.1111/j.1365-4632.2007.03411.x

15. Zouboulis CC. Acne and sebaceous gland function. Clin Dermatol. 2004;22(5):360-6. http://dx.doi.org/10.1016/j.clindermatol. 2004.03.004 